\title{
Aprendizajes sobre el riesgo reputacional en época de Covid-19: la desinformación como riesgo corporativo
}

\section{Learning about reputational risk in the era of Covid-19: disinformation as corporate risk}

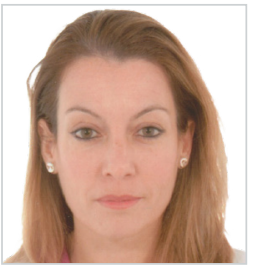

Magdalena Mut Camacho. Doctora en Comunicación empresarial e institucional por la Universitat Jaume I y licenciada en Ciencias de la información por la Universidad Autónoma de Barcelona. Profesora del Departamento de Ciencias de la Comunicación y del Máster Oficial en Nuevas Tendencias y Procesos de Innovación en Comunicación de la Universitat Jaume I de Castelló. Es codirectora, junto a Joan Costa, del Máster Internacional de Dirección y Gestión de la Comunicación para Latinoamérica. Actividad docente e investigadora en la gestión intangible de la organización y en la comunicación corporativa. Ganadora del I Premio Prat Gaballi del Colegio de Publicitarios y Relaciones Públicas de Cataluña. Investigadora del grupo de investigación ENCOM - Engagement and Communication. Investigadora de la Fundación Cotec para la innovación. Es miembro fundador del Foro para el Conocimiento y la Investigación de la Comunicación.

Universitat Jaume I, España

mcamacho@uji.es

ORCID: 0000-0002-1456-8567

Recibido: 04/05/2020 - Aceptado: 23/11/2020

Resumen:

Cuando los profesionales de la comunicación aún se preguntaban cómo deben las empresas sobrevivir y prosperar en la era de la hipertransparencia, teniendo controlados todos los riesgos reputaciones, ahora sobreviene una situación inesperada que alienta dos problemas que todavía se estaba dirimiendo cómo integrarlos en la gestión de la reputación corporativa. Por un lado, lograr recoger el riesgo reputacional en la arquitectura de riesgos corporativos; y por otro, enfrentarse a los riesgos que genera la desinformación, como nuevo contratiempo para la reputación corporativa. Este trabajo indaga sobre la planificación en la empresa española de los efectos de la desinformación mediática en la reputación corporativa, y su concepción como un riesgo corporativo a incluir con el resto de riesgos reputacionales. De forma que, comprobaremos hasta qué punto la empresa española asume el peligro de la desinformación y lo considera un nuevo riesgo reputacional que puede ser más urgente y grave cuando sobreviene una situación como la alerta por pandemia mundial. La principal conclusión es que los riesgos derivados de la desinformación confirman la brecha entre la percepción profesional y las prioridades de la organización.

Palabras clave:

Reputación corporativa; riesgo reputacional; fake news; desinformación; Covid-19.
Received: 04/05/2020 - Accepted: 23/11/2020

\section{Abstract:}

When communication professionals were still asking themselves how companies could survive and prosper in the era of hyper-transparency while keeping all reputational risks under control, an unexpected situation appeared that is now generating two problems that were still in the process of being addressed in terms of how to integrate such risks into corporate reputation management. One difficulty is incorporating reputational risk into the corporate risk architecture; the other involves addressing the risks induced by disinformation, which is a new setback for corporate reputation. This study investigates the planning that takes place in Spanish companies regarding the effects of media disinformation on corporate reputation and the perception of such disinformation as a corporate risk to be included among all other reputational risks. As such, our intention is to confirm the extent to which Spanish companies accept the danger of disinformation, and the degree to which they consider the latter to be a new reputational risk that may be more urgent and serious when a situation such as the current worldwide pandemic occurs without warning. The main conclusion is that the risks related to disinformation reinforce the gap between professional perception and organisational priorities.

Keywords:

Corporate reputation; reputation risk; fake news; disinformation; Covid-19.

Cómo citar este artículo:

Mut Camacho, M. (2020). Aprendizajes sobre el riesgo reputacional en época de Covid-19: la desinformación como riesgo corporativo. Doxa Comunicación, 31, pp. 19-39.

https://doi.org/10.31921/doxacom.n31a1 


\section{Introducción}

Como señala Xifra (2020) es en estos momentos de incertidumbre e inestabilidad cuando la gestión eficiente del riesgo reputacional se ha transformado en una oportunidad para añadir valor intangible a la organización y para mantener la confianza de los públicos.

El riesgo es el efecto de la incertidumbre sobre los objetivos. Bauman dijo, "si no existe una buena solución para un dilema, si ninguna de las actitudes sensatas y efectivas nos acercan a la solución, las personas tienden a comportarse irracionalmente, haciendo más complejo el problema y tornando su resolución menos plausible" (La Vanguardia, 2017). Esta condición encuadra el nuevo rumbo de la sociedad donde no son los hechos, son las emociones las que determinan comportamientos y actitudes sobre una situación, dando como resultado una nueva realidad. Así, nos encontramos de lleno en la era de la posverdad, caracterizada por el auge exponencial de las tecnologías digitales que han hecho florecer canales de información por donde circulan fake news (noticias falsas difundidas con apariencia de verdaderas) que menoscaban los principios de honestidad y veracidad. Este hecho inaugura un nuevo prisma con el que mirar la actualidad y establecer nuevas pautas de relación entre los diferentes actores con los que la empresa se relaciona.

La reputación es uno de los valores más prometedores de la gestión empresarial. Las organizaciones con una buena reputación poseen la confianza de sus públicos. La teoría sobre la reputación corporativa ha alcanzado un nivel de compresión y manejo completamente afianzado en el mundo empresarial y académico. Sin embargo, comprobamos cómo el concepto reputación es tan dinámico que ha de ser vigilado continuamente y mejorado, ya que va virando y modificándose conforme la sociedad vira y se transforma. Ajustado a una realidad que se presenta repentinamente en forma de alerta mundial por pandemia y hunde la economía de los países. La reputación no responde a un concepto teórico inalterable y monolítico, ha de ir trabajándose con mirada poliédrica y visión estratégica, pensando en la organización y su entorno (Mut-Camacho, 2011).

Parte importante de la percepción de los públicos sobre la reputación de una empresa depende de las tareas que esta haga con su comunicación. Hay investigación reciente que expone la incapacidad de las empresas para adaptarse a los avances tecnológicos. Las empresas van por detrás de la sociedad en el uso de los medios sociales para la conversación con sus públicos y la generación de vínculos positivos con su entorno (Aced-Toledano y Lalueza-Bosch, 2016; Costa-Sánchez y Túñez-López, 2019).

En este sentido, la era digital ha sacudido la manera de entender la organización y las relaciones de esta con sus públicos. El ámbito empresarial que poco a poco va adaptándose a los nuevos canales comunicativos, no es inmune a los nuevos peligros del universo comunicativo digital. Las formas de la desinformación mediática son variadas: fake news, bulos, medias verdades, shallowfakes, astroturfing, redes de seguidores falsos, deepfakes, publicidad dirigida, trolling, memes visuales, cibercensura. A todas está expuesta la empresa y se pueden convertir en un riesgo reputacional que debe contemplarse en el manejo de los valores intangibles de la compañía.

Por esta razón, este trabajo busca poner el foco en el punto en el que se cruza la incertidumbre, la desinformación y la reputación corporativa, analizando si considerarlo un riesgo reputacional ayudará a las empresas a reducir la incertidumbre en un mundo cada vez más disruptivo tras la pandemia, y participar de este modo en una correcta planificación de 
la reputación corporativa, ya que las relaciones con los clientes, la sociedad, la administración, los líderes de opinión, los accionistas, los empleados son algunos de los vínculos que se pueden ver dañados con una crisis reputacional derivada de una noticia falsa o información intoxicada.

\section{El riesgo reputacional y sus consecuencias}

En estas circunstancias de alerta sanitaria y confinamiento por pandemia, las empresas han añadido entornos nuevos con los que lidiar. Resulta interesante recuperar el estudio Global Risk Management Survey 2019, donde el riesgo por pandemia o crisis de salud salió en el lugar número $60^{\circ}$. El top mundial de riesgos era hasta hace nada: en primer lugar, sufrir ataques cibernéticos y violación de datos; en segundo lugar, los riesgos de las tecnologías disruptivas; y, en tercer lugar, no saber innovar ni satisfacer las necesidades de los clientes (AON, 2019). En verdad, estos posibles riesgos top siguen estando ahí, pero ahora tienen una perspectiva diferente y su riesgo latente se ha incrementado. Esto es debido a que el riesgo reputacional es un riesgo amplificador que se superpone o se agrega a otros riesgos, especialmente a los riesgos ambientales, sociales y de buen gobierno, e incorpora implicaciones negativas a la materialización, duración o expansión de esos otros riesgos que afectan a la organización, persona, producto o servicio (Llorente \& Cuenca, 2019).

En ese momento, antes de la pandemia mundial, el estudio de la consultora AON pensaba en riesgos reputacionales como el escándalo de las emisiones de Volkswagen, los sobornos de Petrobrás, los sobornos de Wal-Mart, la vinculación al narcotráfico del Grupo Continental, los efectos medioambientales de BP, las afirmaciones de Trump sobre Huawei, el compromiso social de las empresas de moda, etc. Ahora, la crisis recorre todos los estamentos sociales, políticos, económicos y científicos preguntándose cómo será la vida después del Covid-19.

Las consecuencias de esta crisis mundial afectarán al funcionamiento de las empresas, se enfrentan a un impacto en la economía global y en los mercados financieros comparables a la crisis de 2008 (Mckinsey \& Company, 2020) sino mayores (Portillo, 2020). En este sentido, el impacto económico será sin precedentes en España, tan dependiente del sector servicios y abundancia de Pymes, lo que hará su recuperación más difícil (Corporate Excellence, 2020).

Mientras, podemos observar qué está pasando y qué podemos aprender de ello para incrementar el conocimiento sobre la reputación corporativa de las organizaciones y empresas.

Las organizaciones vienen ocupándose de la gestión de riesgos corporativos generalmente a través del consejo de administración, su dirección y restante directiva implicada. La ordenación del riesgo recoge las estrategias de toda la empresa y diseña un plan para identificar eventos potenciales que puedan afectar a la organización, formalizar sus riesgos dentro del riesgo aceptado y proporcionar una seguridad razonable sobre el logro de los objetivos (COSO, 2017). Para las empresas, uno de los riesgos más importantes y más confuso es el riesgo reputacional, hace años que se alienta a las empresas a prepararse para tiempos turbulentos en los que la gestión tradicional de riesgos y crisis deben adaptarse a las nuevas formas del riesgo reputacional (Bonime-Blanc, 2018), sobre todo los ligados al mundo digital (KPMG, 2019).

La importancia de un buen manejo del riesgo está en relación con otros riesgos de la organización, además de la derivada repercusión mediática. Es esencial contar con un programa que recoja todas las probabilidades de suceso y proceso del 
riesgo reputacional, para evitar que la situación derive en un fenómeno complejo y crítico. No hacerlo así, los efectos de una mal resuelta crisis reputacional son (Vizcaíno-González, 2010):

- Pérdida de confianza y lealtad de los empleados

- Reducción de la satisfacción del consumidor

- Coste de capital más elevado

- Mayor volatilidad de los resultados de la empresa

- Mayor dificultad para hacer frente a la crisis

- Incapacidad de explotar las oportunidades de una buena gestión de los valores intangibles.

Es evidente que el impacto en la reputación de una crisis es a largo plazo, a nivel cuantitativo y a nivel cualitativo. Bonime-Blanc habla de tres posibles consecuencias de un riesgo reputacional cuyos efectos serán variables en función del nivel de preparación previa de la organización y de sus líderes:

- 'Golpe mortal', cuando las consecuencias de un riesgo mal gestionado son devastadoras para las personas, los productos, los beneficios y/o el rendimiento. No existe recuperación posible. En estos casos encontramos Lehman Brothers o WordCom

- 'Golpe recuperable', cuando es posible regenerarse además de recuperarse. En este tipo se encuentra Siemens que superó un caso de corrupción y es ejemplo en la implantación de un programa corporativo global anticorrupción.

- 'Caso de mejora de la reputación', cuando una situación sobrevenida deriva en una crisis de la que hay que salir de forma magistral, rápida, responsable y sistemática.

Administrar el riesgo reputacional no es lo mismo que trabajar la reputación. El riesgo requiere una planificación de la incertidumbre de forma exhaustiva, a largo plazo e integral. Bonime-Blanc (2017), plantea que estamos ante un riesgo estratégico clave que todos los consejos deberían supervisar, pero pocos lo hacen. De ahí surge gran parte de la desconexión con los públicos y los problemas vinculados cuando golpea una crisis a una organización.

El riesgo de reputación se define como un riesgo amplificador pues se une a otros riesgos, especialmente a los riesgos ambientales, sociales y de dirección, agregando nuevos problemas al existente. De forma que se une al otro riesgo, lo amplifica y lo acelera, sobre todo cuando la organización no está preparada, no maneja sus riesgos subyacentes y no está adaptada para lidiar con ellos cuando llega la inevitable crisis.

Sin ir más lejos, durante el confinamiento por Covid-19, la Organización Mundial de la Salud (OMS, 2020) advirtió sobre una importante amenaza que acompaña a la pandemia: la infodemia o sobreabundancia de información, de rumores, bulos y datos falsos que propaga la desinformación entre la sociedad.

Contar con una buena comunicación no es suficiente, las empresas deben anticipar las demandas que se pueda suscitar en los reguladores, en los clientes, en los empleados y en la sociedad. Los flujos informativos son clave, pero el éxito dependerá de la reflexión que cada organización sepa hacer de sí misma (PWC, 2017) y de tener un plan que implique a un equipo interdisciplinario y transversal a toda la organización (AON, 2019). La conducción del riesgo reputacional tendrá 
éxito si está coordinado con el resto de riesgos corporativos, ya que todos tienen un impacto sobre la reputación, la marca, las ganancias y el capital (Vizcaíno-González, 2010). Para las organizaciones, medir su reputación corporativa ya era una tarea complicada (Xifra, 2020), intentar calcular sus riesgos reputacionales es más complejo.

\section{La desinformación en el entorno digital como riesgo reputacional}

El auge del fenómeno de la desinformación y la manipulación mediática viene de unos pocos años atrás, cuando en noviembre de 2016 el diccionario Oxford la nombró palabra del año, o cuando la revista Times dedicó su portada de abril a la posverdad con el título Is truth dead? en 2017, o el guiño del Washington Post al cambiar su eslogan de cabecera a Democracy Dies in Darkness.

Las tecnologías de la comunicación han sido las facilitadoras de la transmisión de mentiras e información manipulada, y de que tengan más visibilidad que nunca pues multiplican la mentira exponencialmente (McIntyre, 2018). Es su viralidad, gracias al canal y a la forma del contenido del discurso falso, su gran potencia de ataque. Según un estudio del Massachusetts Institute of Technology, la mentira en Twitter tiene un 70\% más de probabilidad de ser retuiteada. Su propagación es más rápida, más potente y más extensa. El motivo del éxito de su viralidad, en contraposición con la forma de la verdad, es su contenido novedoso y emocional (Vosoughi, Roy y Aral, 2018).

La empresa está expuesta a este entorno de público hiperconectado que consume una inmensa cantidad de información, donde las personas no sólo producen y acceden a contenidos de forma inmediata, sino que consumen y difunden información de manera inmediata. A ello se añade que el aumento en el uso de internet para informarse en fase de pandemia ha crecido en tiempo de conexión con los dispositivos en todas las categorías (AIMC, 2020).

Imagen 1. Porcentajes de aumento de las conexiones a internet durante el confinamiento en España

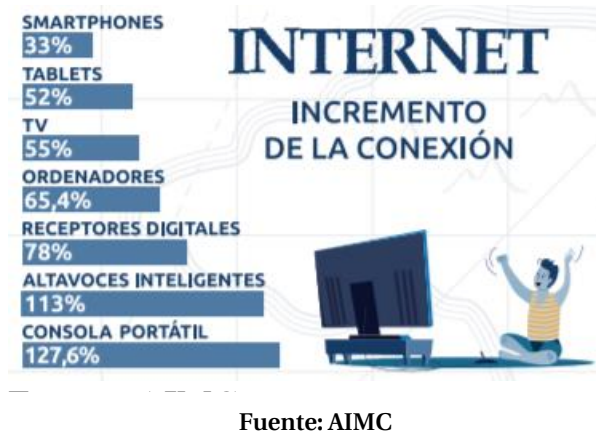

El continuo incremento de los riesgos reputacionales, potenciados por la hiperconexión y las fake news, fueron tratados abiertamente en el Foro Económico Mundial de Davos (2020a), donde se avalaba la necesidad de observar el riesgo reputacional de la desinformación y las fake news como una exigencia inaplazable. Además, los representantes del orden económico y político mundial anunciaron que la próxima ola de tecnologías de la 4a Revolución Industrial cambiará 
drásticamente las economías y sociedades con consecuencias no deseadas. Los ataques cibernéticos se han convertido en un peligro común para las personas y las empresas, por lo que se clasifican como el séptimo riesgo más probable, el octavo más impactante y el segundo riesgo más preocupante para hacer negocios a nivel mundial en los próximos 10 años (World Economic Forum, 2020b: 60).

El Eurobarómetro examinó en qué medida las noticias falsas son percibidas por los ciudadanos de la Unión Europea como un problema para su país y para democracia en general, concluyendo que existe una postura generalizada en toda Europa de hallarnos ante un problema importante. En España el 51\% de los encuestados lo considera un problema muy grave, por encima de la media europea que está en el $44 \%$ de la población. Junto con el $37 \%$ que lo considera un problema grave, se alcanza un total de un $88 \%$ de población española preocupada por el grado de peligrosidad de la desinformación y la manipulación (Comisión Europea, 2018).

Las fake news aumentaron en 2017 un 365\%, generando graves daños reputacionales a instituciones y compañías (Canvas, 2018). Las soluciones que se proponen se dirigen a los dos actores implicados, en primer lugar, los propietarios de las redes sociales, pidiéndoles abandonar su postura de mediadores y se hagan responsables del contenido que publican y comparten sus usuarios, así como de su seguridad y privacidad. La segunda pone el acento en las empresas y sus directivos para que lideren el cambio (Corporte Excellence, 2018). La Comisión Europea ha promovido entre empresas la firma de un Código de Buenas Prácticas sobre la Desinformación (2018). El propósito de este código es unificar acciones que los firmantes pueden implementar para abordar los desafíos relacionados con la desinformación. Entre las medidas que el documento propone se plantea suspender los ingresos publicitarios de quienes adulteren información, cerrar cuentas falsas, identificar claramente la propaganda política, transparencia y verificación de contenidos, etc.

\section{La desinformación, un desafío para la reputación corporativa}

Recordemos que la reputación corporativa es confianza en la organización, el Trust Barometer de Edelman (2019a) alertaba de que la sociedad confía cada vez menos en las organizaciones. Además, el informe advierte del problema social al que nos enfrentamos al revelar que al $73 \%$ de la ciudadanía le preocupa que la desinformación, las noticias falsas y los bots digitales sean un arma moderna de propaganda negativa. No existe una solución clara a la verificación de los hechos que permita discernir de forma rápida qué es real y qué es falso, en consecuencia, la confianza en los negocios a nivel mundial, ronda el 56\%. Y, por tanto, las empresas deben luchar por encontrar la mejor manera de abordar las noticias falsas y las campañas de desinformación que son tan complejas e invasivas (Edelman, 2019b).

Los analistas y consultores en comunicación, así como las empresas consultoras en riesgos corporativos llevan tiempo trabajando la disección de una crisis de desinformación. Estas han enfocado la materia y sus consecuencias desde la perspectiva profesional, conscientes de que han surgido nuevas formas de relación con la opinión pública y de que se han consolidado los medios alternativos de información. Mientras, los canales tradicionales han perdido peso frente al auge de nuevos canales de comunicación como Youtube, Whatsapp, Telegram, Facebook Chat, Twitter, Instagram, entre los más usados. La nueva comunicación supone una oportunidad y un riesgo, "un mero tuit puede movilizar a masas y provocar resultados impensables hace unos pocos años” (Llorente \& Cuenca, 2018a). 
El mundo profesional reconoce el peligro de las fake news en el ámbito empresarial y se propone el desarrollar programas de identidad digital que promuevan la presencia de los directivos y colaboradores de las compañías en redes sociales (Llorente \& Cuenca, 2018b). La lógica es enfrentar la mentira promovida por perfiles falsos con información veraz promovida por embajadores reales e informados. Las fake news son creadas para perseguir audiencias, dañar reputaciones o defender posiciones ideológicas. La solución parte de una escucha y reacción ágil, además de una actitud proactiva en el espacio digital para amplificar la versión de la empresa (Goldhammer, 2017; Chang y Olson, 2017; Pwc, 2018; Deloitte, 2019; Llorente \& Cuenca, 2020: 81).

Se propone que la empresa formule una prevención de riesgos con identificación de vulnerabilidades potenciales y que se desarrolle un plan de acción para mitigar los efectos de la propagación de información falsa. Aconsejan aumentar la estrategia en redes sociales para tener una voz fuerte, apostar por tecnologías de monitoreo innovadoras y tener un plan de respuesta preparado para ser eficaces en atajar el riesgo reputacional.

\section{Objetivos}

Tras comprobar que la desinformación se está convirtiendo en una tendencia que está perjudicando a las organizaciones y que el aumento de la desinformación ha sido exponencial en el periodo Covid-19, resulta conveniente realizar un análisis sobre si la empresa española reconoce la crisis reputacional derivada de la desinformación diseminada por el universo digital como una crisis reputación y, por tanto, formalizada entre sus riesgos corporativos. El objetivo general de la investigación se centra en profundizar en el peligro de la desinformación para la reputación corporativa y determinar si forma parte de las prioridades de la empresa en línea con la realidad analizada. El objetivo último es aportar información relevante para adecuar la teoría a la realidad recogiendo las enseñanzas de este periodo extraordinario.

\section{Metodología}

En esta investigación se ha utilizado una metodología de análisis basada en la realización de encuestas online. El estudio se inició en el mes de febrero de 2020 y finalizó en el mes de mayo de 2020. El cuestionario está compuesto por 10 preguntas que se corresponden con los objetivos investigadores marcados. Se ha trabajado sobre censo cerrado con la base de datos de la fundación propietaria. La representatividad del universo es máxima ya que son todos ejecutivos de empresas responsables de reputación corporativa y expertos en la materia. El universo de la investigación han sido los 578 miembros del think tank Corporate Excellence-Centre for Reputation Leadership, una fundación empresarial especializada en reputación que cuenta en su patronato empresas del Ibex 35-. La muestra se fijó en 261 profesionales especializados en reputación corporativa entre los miembros del think tank.

Los datos obtenidos a través del cuestionario se han almacenado y tratado utilizando el programa informático Excel. Las respuestas obtenidas suponen el $48 \%$ del universo, alcanzando un grado de representatividad aceptable. El margen de error muestral es de $\pm 4,5 \%$ con un nivel de confianza $2=95 \%$. 
La distribución de la muestra por sexos es la siguiente: $61 \%$ hombres y 39\% mujeres. Los participantes tienen titulación universitaria, las más mencionadas son Periodismo (43\%), Publicidad y RR.PP. (23\%), Económicas y Empresariales (18\%) o Derecho (11\%), el resto cuentan con másteres en MBA, Marketing y Comunicación Corporativa.

\section{Resultados}

\subsection{Perfil empresarial de la muestra}

La selección de la muestra permite observar cómo las empresas españolas se enfrentan a este tipo de crisis de reputación, tanto desde la visión del profesional como desde la visión interior de la organización.

Los primeros datos extraídos hacen referencia a las características de la muestra. La mayoría de profesionales expertos en reputación seleccionados trabajan en grandes empresas (87\%), muchas pertenecientes al Ibex35, por tanto, hablamos de opinión informada, con gran experiencia y con un volumen de tareas importante con respecto a la gestión intangible de sus organizaciones.

Los sectores de actividad de las empresas participantes son: financiero y asegurador 35\%, industrial y construcción $23 \%$, sector energético 13\%, y en proporciones menores entre el $9 \%$ y el 7\% se reparten en distribución y logística, sanitario y servicios públicos.

El porcentaje es representativo de los sectores económicos españoles.

\subsection{Percepción sobre el riesgo de reputacional originado por la desinformación}

Es importante señalar que antes de la encuesta se consensuaba el término desinformación como varias formas de manipulación mediática como se ha referido anteriormente.

\section{Gráfico 1. Consideración de la desinformación como una amenaza a la organización}

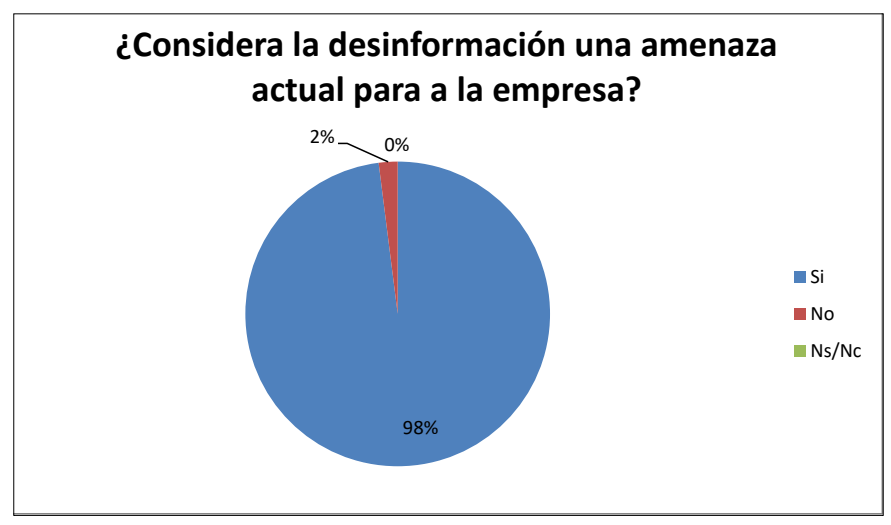

Fuente: Elaboración propia 
Esta es la pregunta más indicativa de hasta que punto los responsables de la reputación en las empresas españolas son sabedores de problema que supone las mentiras y la desinformación, y el nivel de perjuicio para las marcas debido a su viralidad. Es significativo resaltar que nadie muestra ninguna duda, todos los entrevistados tenían una opinión clara y rotunda ya que nadie respondió Ns/Nc (Gráfico 1).

Gráfico 2. Definición y gravedad del problema

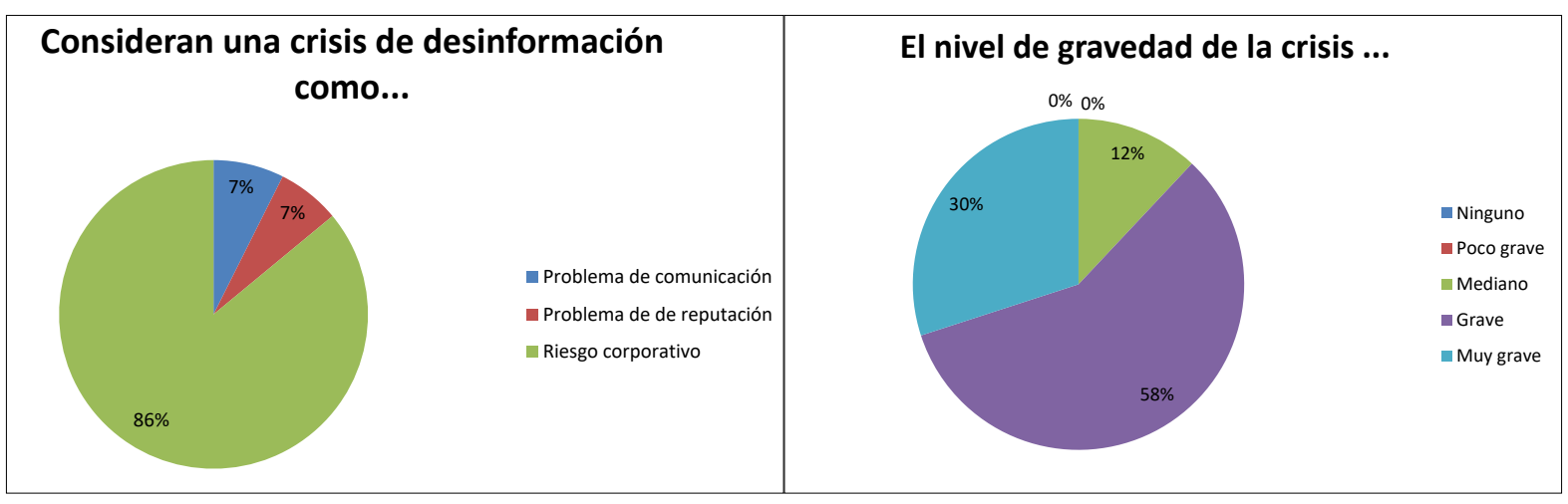

Fuente: Elaboración propia

Es importante recoger que la irrupción de una mentira se considera un ataque al corazón de la organización, un riesgo corporativo que se debe valorar como alarmante y peligroso para el negocio. Esta respuesta es importante para la extracción de conclusiones, ya que, la gran mayoría escoge definir la crisis como riesgo corporativo (Gráfico 2), lo que conlleva una tarea más importante y una función más preeminente. Constituye a su entender una amenaza profunda para la organización. Cuando se les cuestionó sobre el nivel de gravedad de este tipo de crisis, las respuestas se repartieron entre un $30 \%$ muy grave, un $58 \%$ grave y un $12 \%$ gravedad media.

El nivel de gravedad, y por tanto de preocupación, sumando muy grave y grave se alcanza el $88 \%$ de los encuestados. Interesante es observar que nadie respondió ninguna o poca gravedad. 
Gráfico 3. Categoría del riesgo en la arquitectura de riesgos

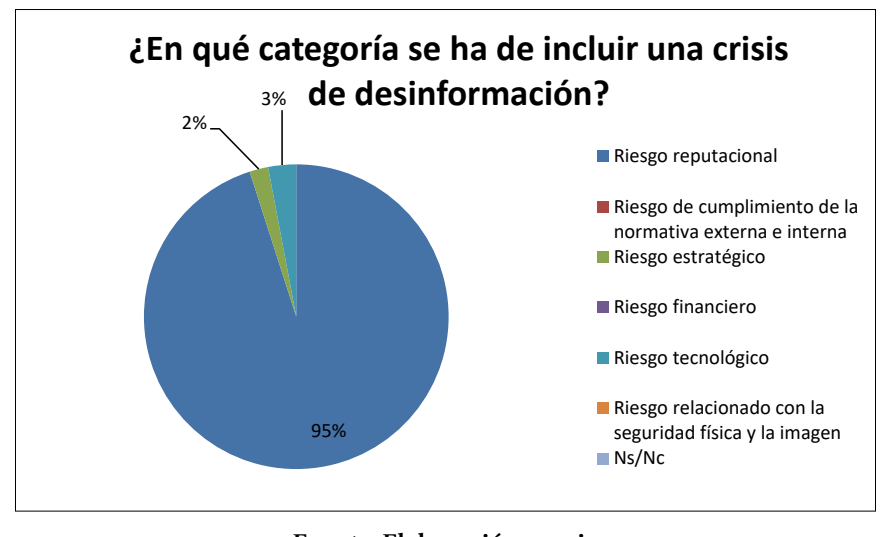

Fuente: Elaboración propia

Por lo que respecta a la categorización del riesgo, entre los profesionales, el más mencionado es el riesgo de reputación corporativa (Gráfico 3), por tanto, afecta a los cimientos sobre los que se construye la confianza de todos los públicos.

\subsection{La empresa española ante una crisis de desinformación}

Gráfico 4. Importancia de este riesgo en el seno de la empresa

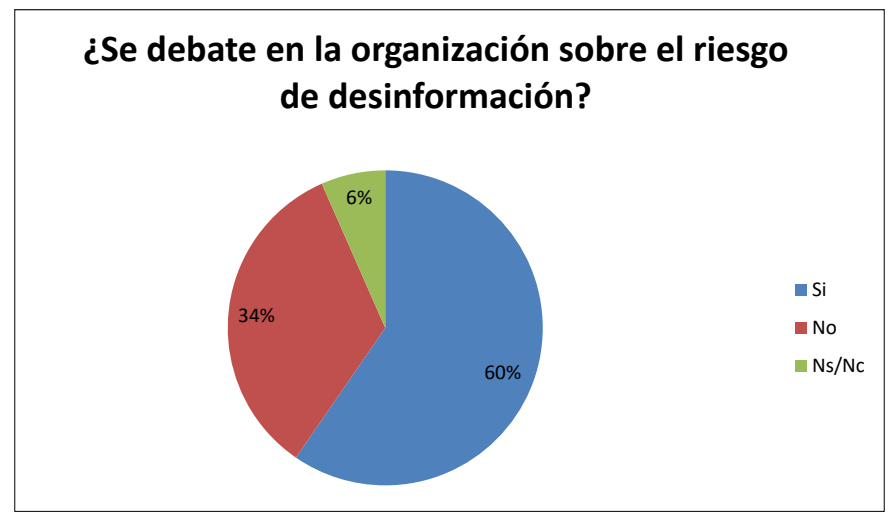

Fuente: Elaboración propia

Por lo que respecta al nivel de debate en el seno de la organización, nos encontramos que en el $60 \%$ de las empresas se habla sobre el peligro que puede suponer una crisis de desinformación para la reputación empresarial (Gráfico 4). Pero, 
curiosamente, este resultado contrasta con la percepción mayoritaria de los profesionales, donde un 98\% alertaba sobre el peligro real y actual. Aún hay un importante porcentaje de empresas donde no se contempla como un tema prioritario.

Gráfico 5. Nivel de formalidad del riesgo en la empresa

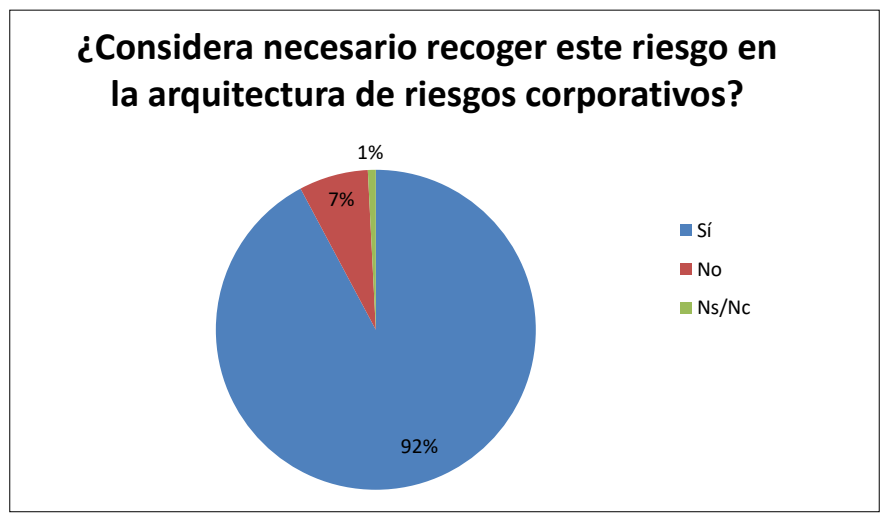

Fuente: Elaboración propia

Los datos evidencian, de forma rotunda, la urgencia de que esta tipología de crisis esté recogida formalmente entre los riesgos corporativos (Gráfico 5). Conviene definirla y trabajarla desde los niveles superiores de la organización al incluirla formando parte del riesgo reputacional.

Gráfico 6. El debate interno sobre el riesgo en el seno de la organización

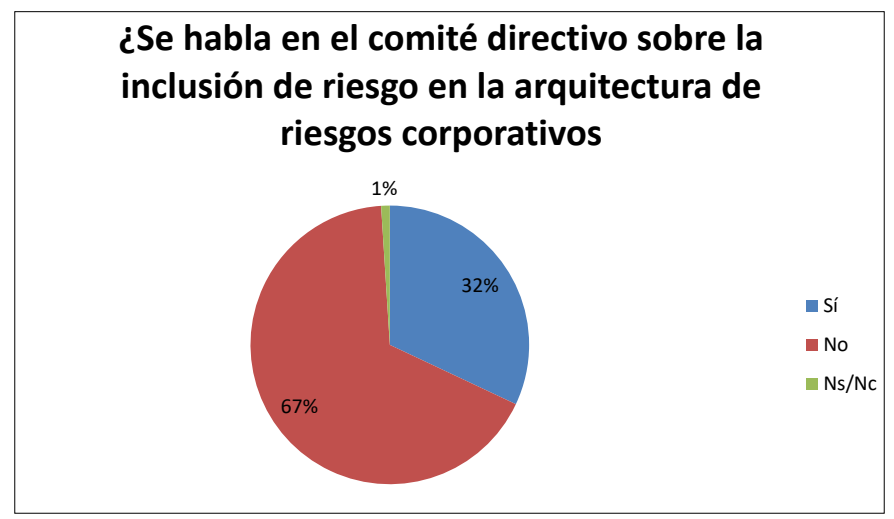

Fuente: Elaboración propia

El análisis de los datos hace posible contrastar la opinión de los profesionales de la reputación corporativa con el interés de la gerencia en este riesgo, estimamos que no es comparable el nivel de prioridad para la organización con el nivel de 
alerta del profesional sobre la alta repercusión reputacional de un ataque a la empresa con una mentira extendida por las redes sociales.

\subsection{Vulnerabilidad de la organización}

Gráfico 7. Área de la empresa más expuesta

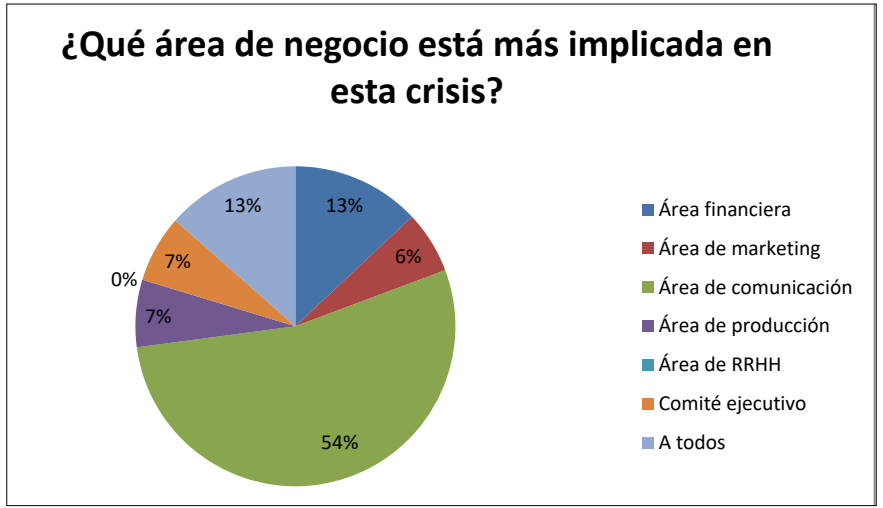

Fuente: Elaboración propia

La medición muestra que, para más de la mitad de los profesionales, el área más vinculada a las tareas de la crisis de reputación vinculada a un bulo es el área comunicación (Gráfico 6). Esto conlleva verificar que el profesional reconoce que es su área de competencias la más adecuada, área que hace años asumió la gestión de la reputación y valores intangibles.

Gráfico 8. Sector vulnerable y actividad vulnerable

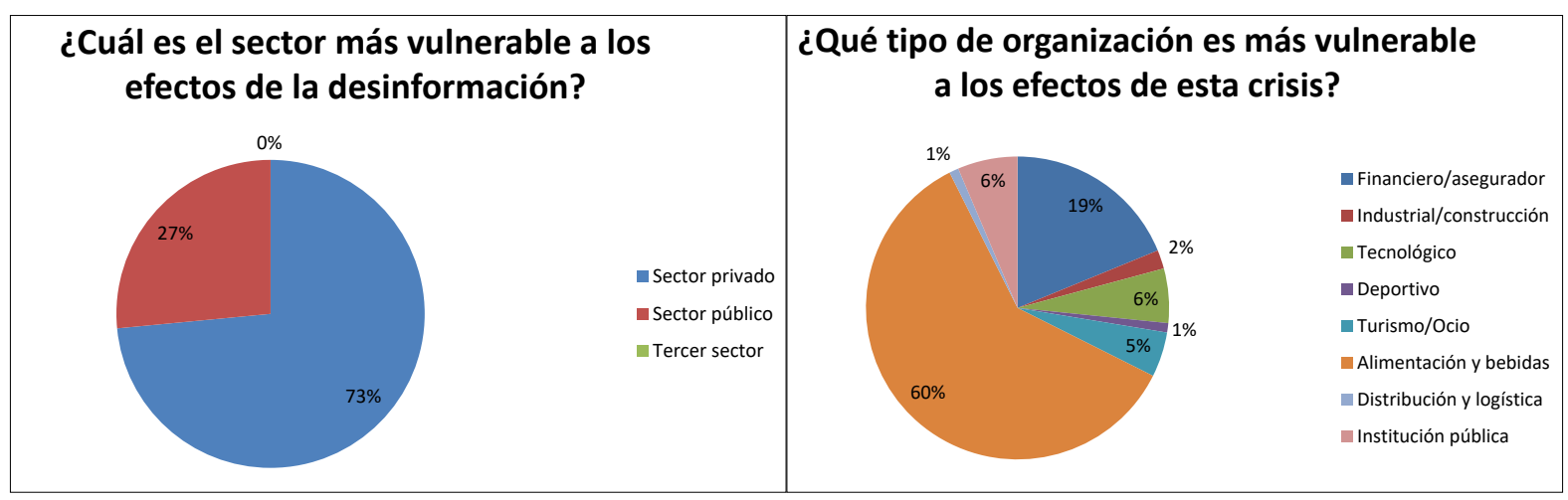

Fuente: Elaboración propia 
Respecto a la percepción de fragilidad y amenaza, esta se acota en el sector privado como el más proclive a ser atacado (Gráfico 7). Un sector donde, por lo general, los efectos de los riesgos son más peligrosos y permanentes como se ha comprobado hasta ahora. Por otra parte, dentro del ámbito de la empresa privada, se estima que el sector de la alimentación es el más expuesto y más vulnerable a un ataque de bulos y falsedades en el universo digital.

Gráfico 9. El valor intangible más vulnerable

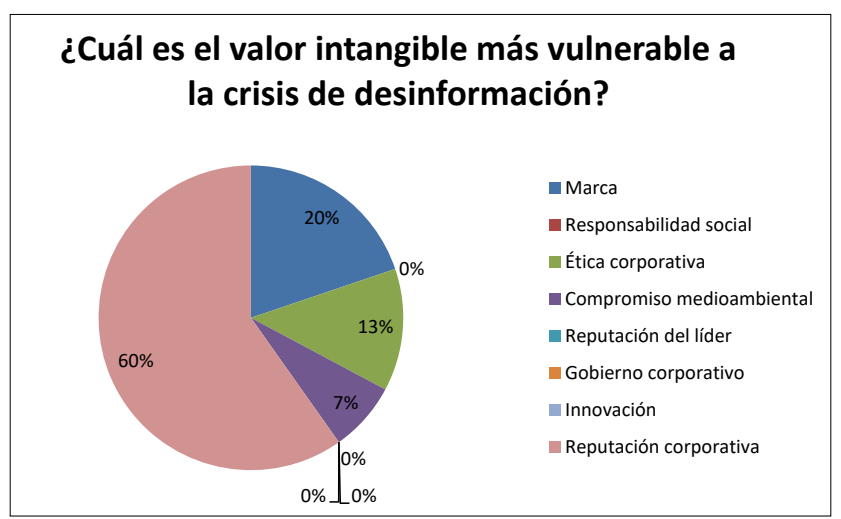

Fuente: Elaboración propia

Este dato refleja que los dos valores intangibles que más se deben proteger son la reputación corporativa y la marca (Gráfico 8). La actitud ética y medioambiental son posturas de la organización que pueden verse atacadas a juicio del profesional. No obstante, a la hora de planificar la prevención de crisis se tendrá en cuenta el profundizar en los efectos a largo plazo en los valores intangibles de la organización. Tanto la reputación del líder, como gobierno corporativo e innovación no se consideran intangibles vulnerables a este tipo de crisis. 


\subsection{Experiencias reales con una crisis de posverdad y sus aprendizajes}

Gráfico 10. Veces que se ha sufrido una crisis de desinformación

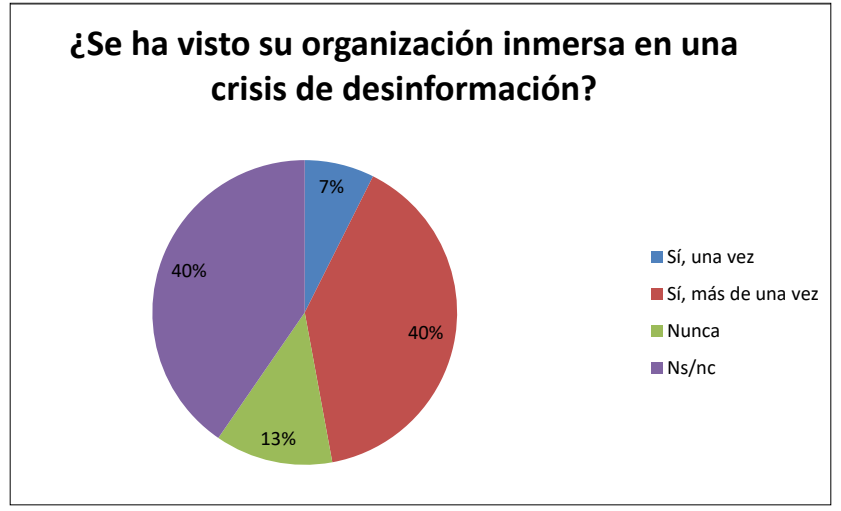

Fuente: Elaboración propia

Preguntados los profesionales sobre este asunto, curiosamente un porcentaje a tener en cuenta (40\%), asegura no saber si le ha sucedido en su organización (Gráfico 9), por lo que se plantean una serie de dudas que no podemos responder. Pensamos que, por un lado, es posible que sea así porque no se encuentra entre las responsabilidades del profesional de la reputación este riesgo reputacional, cuestión que sería grave siendo el experto en esta materia; por otro lado, cabe la posibilidad de que la organización considere esta cuestión crítica como meramente técnica y vinculada a la comunicación digital y, por tanto, no se maneje de forma estratégica sino de forma técnica. Aquí se nos plantean unas dudas que deberían ser resueltas en otra investigación.

Gráfico 11. Aprendizajes de la experiencia

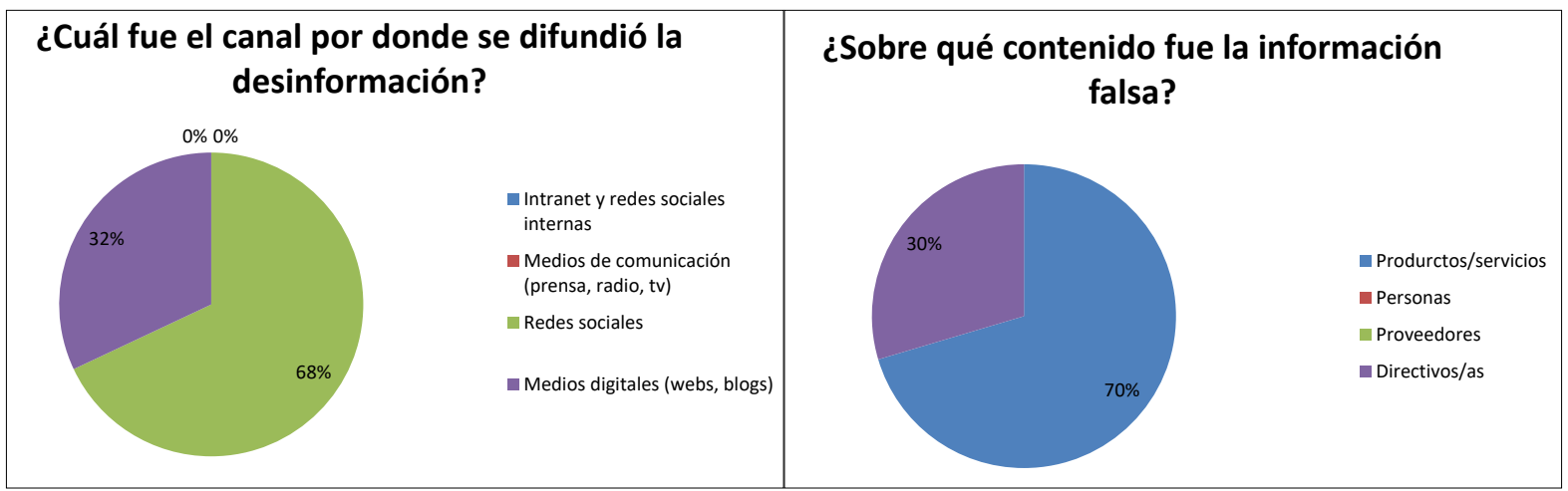

Fuente: Elaboración propia 
Estas respuestas se recogen del $47 \%$ de la muestra que dijo haber sufrido una crisis de desinformación en su organización. Observamos que el principal vehículo de propagación de la mentira fue la RRSS o los medios digitales, corroborándose la realidad de mundo digital en que vivimos. La experiencia relatada muestra que es el producto/servicio el objetivo preferente de los bulos y mentiras que se generan en el universo digital (Gráfico 10).

\subsection{Preparación ante la crisis}

Gráfico 12. Existencia de planes de crisis formalizados

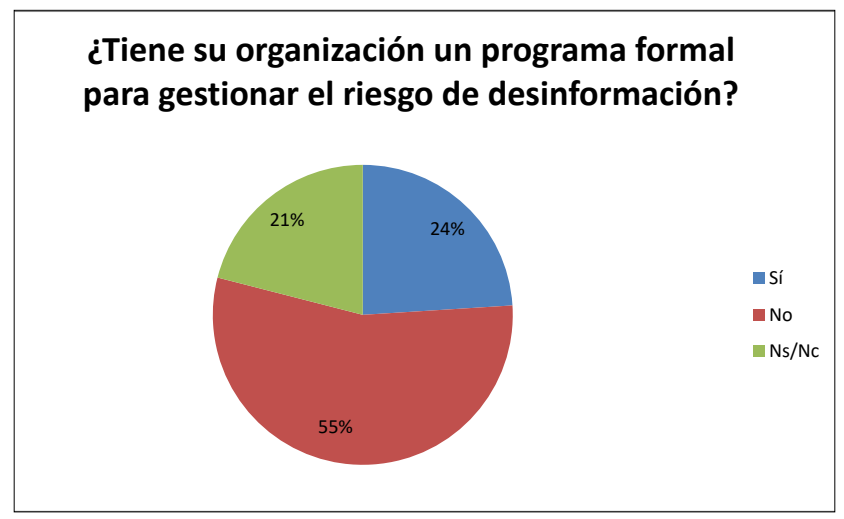

Fuente: Elaboración propia

Si analizamos los datos comprobamos que hay un elevado número de respuestas que llevan a pensar que no existe una preparación para este riesgo (Gráfico 11). Es paradójico que un $21 \%$ no sepa si tienen un plan formal, de nuevo regresamos a las mismas dudas que se originaron en el gráfico 9. 
Gráfico 13. Previsión de la crisis a nivel técnico

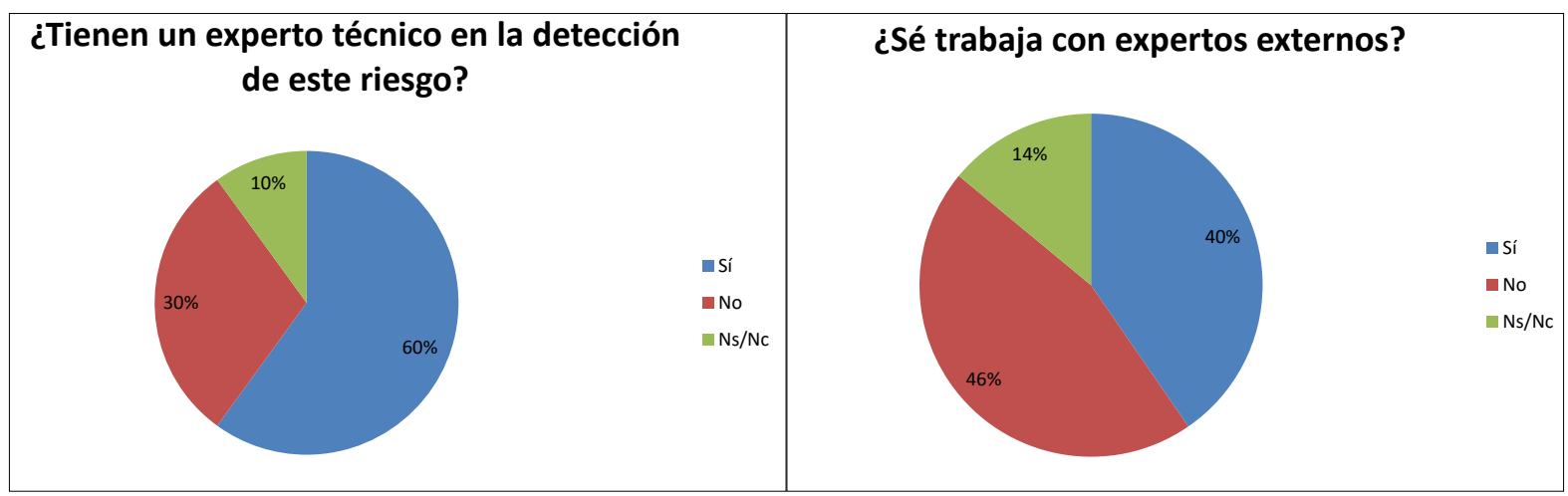

Fuente: Elaboración propia

La existencia en las empresas de perfiles digitales expertos es una evidencia, sin embargo, destaca el $30 \%$ que asegura no tenerlo y el $10 \%$ que no sabe si alguien está haciendo esta tarea (Gráfico 12). Por otra parte, el porcentaje que recurre a ayuda externa se ve por igual repartido entre los que contratan asesoramiento y las que no.

Gráfico 14. Tipo de asesoramiento externo

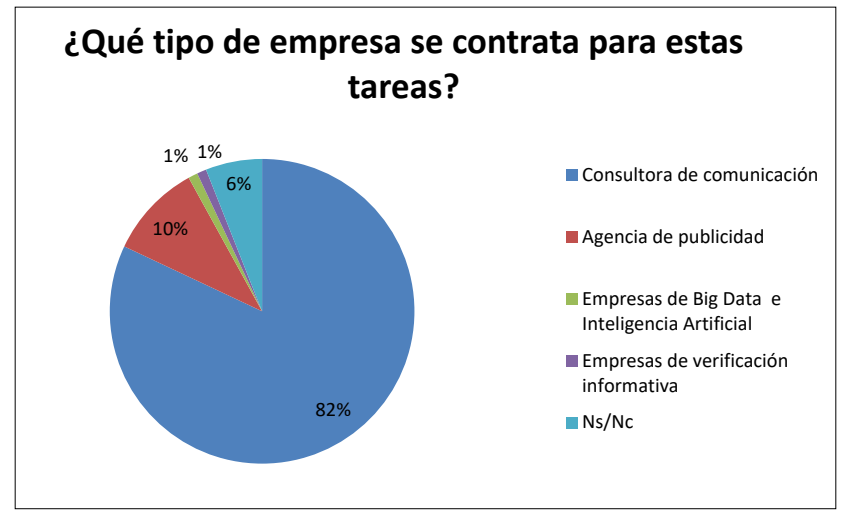

Fuente: Elaboración propia

En este punto comprobamos cómo se recurre, por tradición, en quién la empresa confía para la gestión de cuestiones vinculadas con la reputación corporativa y la comunicación corporativa: la consultora de comunicación. En la actualidad aún no se hace uso de empresas de verificación o IA para tareas de gestión de riesgos de desinformación y manipulación mediática. 


\subsection{Evolución del riesgo en el futuro}

Gráfico 15

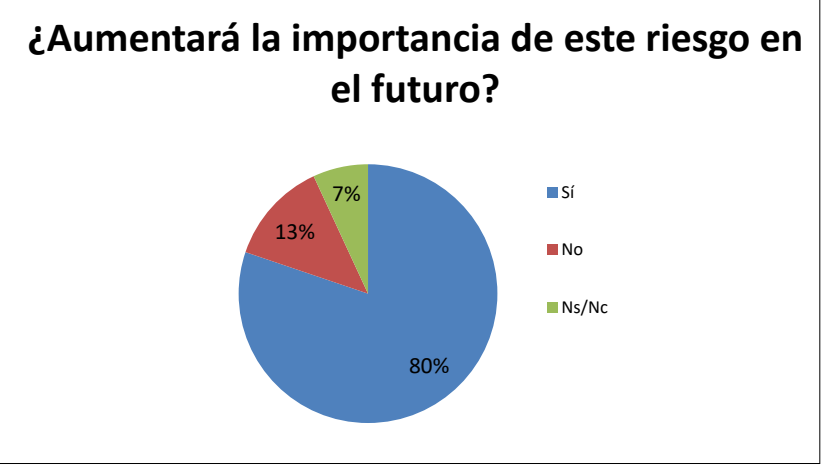

Fuente: Elaboración propia

Para el profesional de la reputación este riesgo se va a ver aumentado tanto por sí mismo como por ser un riesgo que puede llegar vinculado a otro riesgo con el que la empresa está lidiando.

\section{Conclusiones y discusión}

Los resultados de este estudio confirman que para los profesionales de la reputación existe una amenaza real en la desinformación de las redes sociales e internet, que nos hallamos ante un problema conocido y peligroso, y que lo identifican como riesgo real, grave y urgente. Más si cabe en este periodo que estamos atravesando, lleno de incertidumbres y de dudas sobre el presente y el futuro. De hecho, la percepción de los profesionales está en línea con los informes que las consultoras de estrategia revelan desde hace unos años atrás. Sin embargo, no encontramos el mismo nivel de prioridad en la alta dirección de la empresa, donde este factor reputacional no tiene la misma excepcionalidad e impacto en comparación. Contrasta que, para los participantes, un 92\%, es esencial medir y recoger este riesgo; mientras que, la alta dirección no prioriza esta posibilidad, tan sólo un $32 \%$ de las empresas lo tiene contemplado en su arquitectura de riesgos, y plasmado en un programa de trabajo muy pocas empresas (21\%).

De forma que, determinamos que los responsables de reputación de las empresas españolas son conscientes de que una crisis de desinformación en las redes es un claro riesgo reputacional compartida por casi la totalidad de los profesionales (98\%). Este dato es un importante indicador, si tenemos en cuenta que gran parte de la muestra son grandes empresas, las que se consideran más vulnerables por quienes quieren perjudicar a las marcas. Confirmamos que este es un riesgo a evitar y a tener presente entre los riesgos corporativos con el fin de ayudar a las organizaciones a evaluar y mejorar sus sistemas de control interno de la reputación corporativa. 
Tomando en consideración que ninguna marca está libre de un ataque de desinformación y que los profesionales reconocen estar ante un grave riesgo reputacional, se asume que la empresa enfrentada a una fake news puede ver desequilibrada su reputación y toparse con tareas importantes de incierto resultado, porque dentro de la manipulación mediática actúan frentes diversos y la crisis es novedosa y falta experiencia.

El hecho de que a las personas les cueste decidir si se encuentran ante una noticia falsa o verdadera, hace necesario para la empresa vigilar lo que dice y lo que otros dicen de ella, el control y monitoreo del universo digital de Internet es una recomendación de las empresas consultoras investigadas.

Por tanto, es la anticipación la solución que se vislumbra ante el riesgo reputacional de la desinformación y las fake news, además de la construcción del sólido relato corporativo mantenido en el tiempo sobre una fuerte reputación corporativa y una buena comunicación, utilizando los medios sociales de forma eficiente y creativa para alcanzar el grado de confianza que haga desactivar o atenuar la desinformación y la mentira.

El cambio en el comportamiento de la ciudadanía en el actual periodo de confinamiento con nuevas demandas, valores, percepciones y preocupaciones respecto a las marcas, debido a diferentes causas como la transformación tecnológica, social, cultural y el hecho de que la digitalización ha empoderado al consumidor con nuevas herramientas, más que nunca es la gestión de la reputación una tarea estratégica. Ese cambio de comportamiento del ciudadano tiene en las fake news su más peligrosa manifestación, como alertaba la OMS. La doble cara de la mentira, por un lado, la facilidad para sembrar el desprestigio de quien desea perjudicar a la empresa, y por otro, la facilidad para caer en la trampa por parte del receptor, obliga a la empresa a observar el problema como una situación de gran dificultad, ya que muchos de los ingredientes de la desinformación son incontrolables. Sin embargo, también se vislumbra luz pues el consumidor se hace consciente y deslegitima al hater poniendo en duda todo lo negativo que se dice sobre una empresa en redes. Sin embargo, cabe señalar que esto sucede en circunstancias normales, puesto que en excepcionales e insólitas hemos comprobado cómo se puede creer cualquier noticia que se viraliza o declaraciones en medios de comunicación.

Las circunstancias que enfrentamos muestran que la lucha contra la desinformación es urgente y compleja. Las facilidades con las que se esparcen las fake news va en contra de las empresas, ya que, según hemos comprobado, las personas prefieren informarse sobre una marca con los amigos y familiares. Es decir, el entorno más cercano es el más confiable, ganando en confianza a lo que las organizaciones dicen sobre sí mismas. Esta situación añade un doble problema para las organizaciones, pues se confía menos en lo que la empresa dice de sí misma, y las fake news se nutren del método de la recomendación en su viralidad; en consecuencia, la lucha contra la desinformación es inaplazable, más cuando el aumento de las noticias, recomendaciones y cuestiones varias han aumentado en las redes sociales e internet en confinamiento.

Los resultados revelan que las empresas que se han visto afectadas por una crisis de reputación derivada de la desinformación, el bulo se propagó por redes sociales e internet, convirtiéndose en el lugar propicio para alimentar bulos. Las áreas intangibles más vulnerables son la marca y la ética o responsabilidad.

Se aconseja cultivar y mantener una reputación buena y sólida. Esto será un escudo ante una crisis de reputación pues, incluso en la era de la desinformación, la autenticidad ocupa el primer puesto en la escala de valores de las personas. La 
capacidad de transformación social de las empresas dependerá de su capacidad para mantenerse fieles a sus valores, ser transparentes y adaptarse a los cambios.

Por otra parte, la investigación recoge unas dudas y un atisbo. Las dudas surgen a tenor de las respuestas sobre las experiencias con este tipo de crisis. Muchos de los profesionales mostraban desconocimiento sobre el suceso o su gestión, por lo que juzgamos que es extraño encontrar que una crisis que afecta a la reputación corporativa no sea tratada por el experto.

El atisbo parte, por un lado, de las externalizaciones de la empresa para vigilar o solucionar el conflicto y, por otro, de la estimación a futuro de los profesionales, consideramos que se abre un área de especialización profesional distinta y nueva que requerirá de expertos en estas crisis.

En consecuencia, corroboramos que la realidad muestra un verdadero riesgo reputacional. El profesional de la reputación de las organizaciones españolas está preocupado por los efectos de la desinformación en internet y las redes sociales, y considera que es una prioridad en la gestión de la reputación corporativa. Podemos concluir que un nuevo riesgo se ve asociado al concepto reputación corporativa. Las organizaciones pueden evitar riesgos y aprovechar el conocimiento de este riesgo para potenciar sus procesos y un enfoque holístico como parte de sus esfuerzos de comunicación, si se marcan este asunto como una prioridad.

\section{Referencias bibliográficas}

Aced-Toledano, Cristina; Lalueza-Bosch, Ferran (2016). “¿Qué contenidos publican las empresas en los medios sociales? Análisis del discurso de las compañías del IBEX 35 y del Fortune 500 en blogs corporativos, Facebook y Twitter”. Revista Internacional de Relaciones Públicas, v. 6, n. 11,135-154.

AIMC Asociación para la Investigación de Medios de Comunicación (2020). AIMC Cuaderno de Bitácora, 16 de abril. Disponible en: https://www.aimc.es/otros-estudios-trabajos/aimc-cuaderno-bitacora-estudio-medios-la-espana-confinada/ resultados-aimc-cuaderno-bitacora/

AON (2019). Managing Risk: How to maximize performance in volatile times. Disponible en: https://www.aon. com/2019-top-global-risks-management-economics-geopolitics-brand-damage-insights/index.html

Bonime-Blanc, Aandrea (2018). Manual de riesgo reputacional. Madrid: Corporate Excellence-Centre for Reputation Leardership.

Canvas (2018). Approaching the Future 2018. 20 de abril. Disponible en: https://canvasconsultores.com/wp-content/ uploads/2018/06/Approaching\%20the\%20Future\%202018_.pdf

Chang, Benjamin; Olson, Tom (2107). Fake News: A Threat or Business as Usual? Burson-Marseteller, 27 de julio. Disponible en: http://www.burson-marsteller.com/bm-blog/fake-news-a-threat-or-business-as-usual/

Comisión Europea (2018). EU Code of Practice on Disinformation. Shaping Europe's digital future, 26 de septiembre. Diponible en : https://ec.europa.eu/newsroom/dae/document.cfm?doc_id=54454 
Commisión Europea (2018). Eurobarometro. Fake News and Disinformation Online. Disponible en: https://ec.europa.eu/ commfrontoffice/publicopinion/index.cfm/ResultDoc/download/DocumentKy/82797

Corporate Excellence (2020). Covid-19. Análisis e impacto reputacional: guía para empresas. Disponible en: https://www. corporateexcellence.org/recurso/resumen-ejecutivo-covid-19-analisis-e-impacto/6be05000-e098-81f9-7776-821d33f3e $3 \mathrm{c} 8$

Corporte Excellence (2018). Reputación y riesgo reputacional. Disponible en: https://www.corporateexcellence.org/ recurso/las-fake-news-crecen-un-365-y-amenazan-la/0aec2795-d202-1b03-c239-512f623df7ca

COSO. Committee of Sponsoring Organizations of the Treadway Commision (2017). Guidance on Enterprise Risk Management. Disponible en: https://www.coso.org/Pages/guidance.aspx

Costa-Sánchez, Carmen; Túñez-López, Miguel (2019). Contenidos audiovisuales en social media. Análisis comparativo de Facebook y Youtube. Fonseca, Journal of Communication, v.19, 223-236.

Deloitte (2019). Future of risk in the digital era. Transformative change. Disruptive Risk. Disponible en: https://www2. deloitte.com/us/en/pages/advisory/articles/risk-in-the-digital-era.html

Edelman (2019a). 2019 Edelman Trust Barometer. 20 enero. Disponible en: https://www.edelman.com/ research/2019-edelman-trust-barometer

Edelman (2019b). Business must combat fake news. 20 febrero. Disponible en: https://www.edelman.com/insights/ business-must-combat-fake-news

Foro Económico Mundial (2020). The Gobal Risks Report. An Unsettled World. Disponible en: http://www3.weforum.org/ docs/WEF_Global_Risk_Report_2020.pdf

Gartner (2017). Gartner Top Strategic Predictions for 2020 and Beyond. https://www.gartner.com/smarterwithgartner/ gartner-top-strategic-predictions-for-2020-and-beyond

Goldhammer, Gary (2017). Truth: The New Digital Divide. Hill and Knowlton Strategies: https://www2.hkstrategies.com/ global/en/truth-new-digital-divide/

Kantar. (2019). Dimensión 2019. Disponible en: https://www.kantarmedia.com/dimension/es/ authentic-communication-in-a-mistrusting-world/download-report-authentic

Keyes, Ralph. (2004). Post-Truth era: dishonesty and deception in contemporary life. Nueva York: St. Martin's Press.

KPMG (2019). Vientos de cambio. La gestión de los riesgos en el mundo digital. Disponibel en: https://www.tendencias. kpmg.es/wp-content/uploads/2019/04/Informe_Riesgos_Abril2019.pdf

Llorente \& Cuenca (2018a). Contra las Fake News en la empresa: Real Advocacy. Madrid: Llorente \& Cuenca.

Llorente \& Cuenca (2018b). Uno, no 31. Madrid: Llorente \& Cuenca.

Llorente \& Cuenca. (2019). El riesgo reputacional en los consejos del Ibex 35: Logros y retos. Disponible en: https://ideas. llorenteycuenca.com/2019/09/el-riesgo-reputacional-en-los-consejos-del-ibex-35-logros-y-retos/ 
Llorente \& Cuenca (2020). Disrupción. Madrid: Llorente \& Cuenca.

Llorente, José-Antonio (2019). Comunicar en tiempos de disrupción. UNO (nov.), 9-10.

McIntyre, Lee (2018). Posverdad. Madrid: Cátedra.

Mckinsey \& Company (2020). COVID-19: Implications for business. Disponible en: https://www.mckinsey.com/ business-functions/risk/our-insights/covid-19-implications-for-business

Mut-Camacho, Magdalena. (2011). El director de comunicación del cambio. Revista Interanacional de Relaciones Públicas, vol. 1, n. 2, pp. 107-118.

Portillo, Juande (2020). Cinco Días. Obtenido de El FMI prevé que el PIB de España caiga un 8\%, igualando en un año toda la crisis anterior: https://cincodias.elpais.com/cincodias/2020/04/14/economia/1586865124_506836.html

PwC (2017). Encuesta Mundial de Seguridad de la Información 2018. Disponible en: https://www.pwc.es/es/digital/encuesta-mundial-ciberseguridad-2018/descarga-encuesta-mundial-ciberseguridad-2018.html

PwC (2018). Digital Pulse. The fake news problem (and what to do about it): https://www.digitalpulse.pwc.com.au/ fake-news-problem-business/

RAE (2020). Diccionario de la lengua española. https://dle.rae.es/posverdad

Vosoughi, Soroush; Roy, Deb; Aral, Sinan (2018). The spread of true and false news online. Science, 359(6380), pp. 1146-1151. World Economic Forum (2019). Manifiesto de Davos 2020: El propósito universal de las empresas en la Cuarta Revolución Industrial. Disponible en: https://es.weforum.org/agenda/2019/12/ manifiesto-de-davos-2020-el-proposito-universal-de-las-empresas-en-la-cuarta-revolucion-industrial/

World Economic Forum (2020). The Global Risks Report 2020: An Unsettled World. Disponible en: http://www3.weforum. org/docs/WEF_Global_Risk_Report_2020.pdf

Xifra, Jordi (2020). Comunicación corporativa, relaciones públicas y gestión del riesgo reputacional en tiempo del Covid-19. El profesional de la información, v. 29, n. 2, e290220. 
